\title{
Comparing the properties of the X-shaped bulges of NGC 4710 and the Milky Way with MUSE`
}

\author{
O. A. Gonzalez ${ }^{1,2}$, D. A. Gadotti ${ }^{1}$, V. P. Debattista ${ }^{3}$, M. Rejkuba ${ }^{4,5}$, E. Valenti ${ }^{4}$, M. Zoccali ${ }^{6,8}$, L. Coccato $^{4}$, \\ D. Minniti ${ }^{7,8,9}$, and M. Ness ${ }^{10}$ \\ ${ }^{1}$ European Southern Observatory, Ave. Alonso de Cordova 3107, Casilla 19, 19001 Santiago, Chile \\ e-mail: ogonzale@eso.org \\ 2 Institute for Astronomy, University of Edinburgh, Royal Observatory, Blackford Hill, Edinburgh, EH9 3HJ, UK \\ 3 Jeremiah Horrocks Institute, University of Central Lancashire, Preston PR1 2HE, UK \\ ${ }^{4}$ European Southern Observatory, Karl-Schwarzschild Strasse 2, 85748 Garching, Germany \\ 5 Excellence Cluster Universe, Boltzmannstr. 2, 85748 Garching, Germany \\ ${ }^{6}$ Instituto de Astrofísica, Facultad de Física, Pontificia Universidad Católica de Chile, Av. Vicuña Mackenna 4860, Santiago 22, \\ Chile \\ 7 Departamento de Ciencias Físicas, Universidad Andrés Bello, República 220, Santiago, Chile \\ 8 The Milky Way Millennium Nucleus, Av. Vicuña Mackenna 4860, 782-0436 Macul, Santiago, Chile \\ 9 Vatican Observatory, V00120 Vatican City State, Italy \\ 10 Max-Planck-Institut fur Astronomie, Konigstuhl 17, 69117 Heidelberg, Germany
}

Received 22 November 2015 / Accepted 8 March 2016

\begin{abstract}
Context. Our view of the structure of the Milky Way and, in particular, its bulge is obscured by the intervening stars, dust, and gas in the disc. While great progress in understanding the bulge has been achieved with past and ongoing observations, the comparison of its global chemodynamical properties with respect to those of bulges seen in external galaxies has yet to be accomplished. Aims. We used the Multi Unit Spectroscopic Explorer (MUSE) instrument installed on the Very Large Telescope (VLT) to obtain spectral and imaging coverage of NGC 4710. The wide area and excellent sampling of the MUSE integral field spectrograph allows us to investigate the dynamical properties of the X-shaped bulge of NGC 4710 and compare it with the properties of the X-shaped bulge of the Milky Way.

Methods. We measured the radial velocities, velocity dispersion, and stellar populations using a penalised pixel full spectral fitting technique adopting simple stellar populations models, on a $1^{\prime} \times 1^{\prime}$ area centred on the bulge of NGC 4710 . We constructed the velocity maps of the bulge of NGC 4710 and investigated the presence of vertical metallicity gradients. These properties were compared to those of the Milky Way bulge and to a simulated galaxy with a boxy-peanut bulge.

Results. We find the line-of-sight velocity maps and 1D rotation curves of the bulge of NGC 4710 to be remarkably similar to those of the Milky Way bulge. Some specific differences that were identified are in good agreement with the expectations from variations in the bar orientation angle. The bulge of NGC 4710 has a boxy-peanut morphology with a pronounced X-shape, showing no indication of any additional spheroidally distributed bulge population, in which we measure a vertical metallicity gradient of $0.35 \mathrm{dex} / \mathrm{kpc}$. Conclusions. The general properties of NGC 4710 are very similar to those observed in the Milky Way bulge. However, it has been suggested that the Milky Way bulge has an additional component that is comprised of the oldest, most metal-poor stars, which is not part of the boxy-peanut bulge structure. Such a population is not observed in NGC 4710, but could be hidden in the integrated light we observed.
\end{abstract}

Key words. galaxies: bulges - galaxies: individual: NGC 4710 - galaxies: kinematics and dynamics - Galaxy: bulge

\section{Introduction}

The understanding of galactic bulges is a basic step to unveiling the formation history and evolution of galaxies. By mapping the kinematics, stellar population, and morphological signatures of the bulge of a given galaxy, it is possible to constrain the history of events that occurred during the assembly of the galaxy.

Bulges are generally classified into three distinct groups: I) classical bulges, which are spheroidal components dominated by velocity dispersion of old, alpha-enhanced stars as expected from a fast and early formation via dissipative collapse or mergers (e.g. Brooks \& Christensen 2016, and references therein);

* Based on observations collected at the ESO La Silla-Paranal Observatory within MUSE science verification program 60.A-9307(A).
II) pseudo-bulges, which are disc-like, rotation dominated structures populated by young, metal-rich stars, formed from the inflow of gas to the centre of the galaxy due to the influence of a bar (Kormendy 2013); and III) boxy-peanut (BP) bulges which are the result of buckling bar instabilities that favour the heating of the stellar orbits along the vertical direction resulting in a thick structure that swells up from the disc in a peanut or X-shape ${ }^{1}$ (e.g. Combes \& Sanders 1981; Athanassoula 2005). Common in disk galaxies, BP bulges are found in nearly half of the edge-on

1 We note that whether these structures appear boxy, peanut or $\mathrm{X}$-shaped, depends on projection effects and the strength of the buckling instability. We therefore indistinguishably use the terms BP and $\mathrm{X}$-shape bulge here to refer to same structure, i.e. the vertically thickened inner parts of the stellar bar. 
disc galaxies, with a fraction missed due to unfavourable orientation (Lütticke et al. 2000). Even our own Galaxy has recently been proven to host one (Wegg \& Gerhard 2013, and references therein). However, the stellar population properties of BP bulges have not yet been entirely defined, mostly because of the difficulties of disentangling the different bulge components in the analysed samples. As recently pointed out by Laurikainen \& Salo (2016), dedicated studies of individual galaxies, where both the structural decomposition and stellar population analysis can be performed, is fundamental to characterise the properties of BP bulges.

In the Milky Way, the presence of a bar in the inner regions is now well established (Stanek et al. 1994) and its boxy shape has been mapped by different IR surveys such as the COsmic Background Explorer (COBE), Two Micron All-Sky Survey (2MASS), and most recently the VISTA Variables in the Via Lactea (VVV) ESO public survey (Minniti et al. 2010). The radial velocity measurements from spectroscopic surveys, based on hundreds of velocities of M-giants in the Bulge Radial Velocity Assay (BRAVA; Howard et al. 2009) and K-giants in Giraffe Inner bulge Survey (GIBS; Zoccali et al. 2014), have provided strong evidence for cylindrical rotation, which is the expected kinematical signature of rotating bars (but see Williams et al. 2011). Furthermore, following the boxy shape of the Galactic bar mapped by COBE and 2MASS, the analysis of the distribution of red clump stars from different datasets has provided independent evidence for the X-shaped morphology of the Milky Way (Nataf et al. 2010; McWilliam \& Zoccali 2010; Saito et al. 2011). Recently, Wegg \& Gerhard (2013) used photometric data from the VVV survey to map the X-shaped bulge in detail, thus reaffirming the BP nature of the Galactic bulge. Recent stellar population studies have revealed a dominating old (10 Gyr) $\alpha$-enhanced population and the presence of a radial metallicity gradient (e.g. Zoccali et al. 2003; Gonzalez et al. 2013; Valenti et al. 2013). The presence of young $(<5 \mathrm{Gyr})$, metalrich stars, particularly at low latitudes, has also been discussed (Bensby et al. 2013; Ness et al. 2014; Dékány et al. 2015). It has been suggested that the observed spatial distribution of $[\mathrm{Fe} / \mathrm{H}]$, $[\alpha / \mathrm{Fe}]$, and possibly also stellar ages in the Galactic bulge, could be the result of a contribution of different components dominating at different distances from the Galactic plane, namely the BP bulge and a classical bulge (Babusiaux et al. 2010; Hill et al. 2011; Dékány et al. 2013).

Large new datasets are therefore providing new observational insights into the bulge of our Galaxy. However, a decomposition of the different components of the Milky Way bulge is highly complicated by our location in the disc of the Galaxy as well as a combination of effects due to extinction (Gonzalez et al. 2012), line-of-sight depth and bar orientation (Wegg \& Gerhard 2013), and the overall geometry and kinematics of different overlapping Galactic components. It is then left to a reconstruction method, based on the interpretation of the different sets of observations described above. Image decomposition, on the other hand, can be used to study the different bulge components of other galaxies (e.g. Gadotti 2012; Laurikainen et al. 2005, 2010; Méndez-Abreu et al. 2014). It is for this reason that it becomes fundamental to provide a link between the techniques used in the study of the unresolved stellar properties of external galaxies and those used in the Milky Way (see Gonzalez \& Gadotti 2016, for a recent review on this matter). In this context, NGC 4710 appears as an ideal candidate to test the interpretations of the Milky Way bulge observations.

According to NED and HyperLeda databases, NGC 4710 is an edge-on, early-type (S0 or SA(r)0) galaxy in the nearby universe. It is located in the Virgo cluster and is included in the $\mathrm{ATLAS}^{3 \mathrm{D}}$ sample of 260 early-type (E and S0) galaxies. NGC 4710 is unusually gas rich, has the second highest average CO surface density (Young et al. 2011), and is one of the two galaxies most significantly affected by dust extinction in the sample of Scott et al. (2013). Krajnović et al. (2013) list this galaxy among a sample of 13 "uncertain" galaxies in the early-type ATLAS ${ }^{3 \mathrm{D}}$ sample given the strong dust features in the nucleus and uncertain nuclear profile fits. The ionised, molecular gas and stellar kinematics of NGC 4710 are aligned (Davis et al. 2011) and its stellar $V_{\mathrm{rms}}=\sqrt{V^{2}+\sigma^{2}}$ shows a butterfly-like shape characteristic of galaxies with small bulges (Cappellari et al. 2013).

NGC 4710 can be found among the galaxies sampled by the Spitzer Survey of Stellar Structure in Galaxies $\left(\mathrm{S}^{4} \mathrm{G}\right)$ and the recent morphological analysis of Buta et al. (2015) classified this galaxy as an exactly edge-on barred galaxy with a X-shaped bulge and visible ansae on each side of the centre. Furthermore, no co-existing, large-scale classical bulge component has been found based on its light-decomposition analysis (Gadotti 2012).

Based on the fact that both the Milky Way and NGC 4710 have a BP bulge, it is possible to build a link between the studies of resolved and unresolved properties of stellar populations of this type of bulges, thus bringing to a common ground our detailed knowledge of the Milky Way bulge and that of unresolved external galaxies. With this aim, in this article we investigate the kinematics and stellar populations of the BP bulge of NGC 4710 and we compare them directly to those of the Milky Way bulge. This comparison becomes particularly important when considering the recent study by Dékány et al. (2013), which suggest that the spatial distribution of the oldest population of the Milky Way bulge, traced by RR Lyrae stars, follows a spheroidal component and possibly co-exists with the BP bulge of the Milky Way (but see also Pietrukowicz et al. 2015). Therefore, we can also explore the presence of any specific signature that is not found in the pure BP bulge of NGC 4710, which can therefore be identified as an additional component in the bulge of the Milky Way.

\section{Observations and data reduction}

The observations of NGC 4710 were taken as part of the MUSE science verification observing run in June 2014. MUSE (Bacon et al. 2010) is an optical wide-field integral field spectrograph installed in UT4 at the ESO Very Large Telescope. This instrument uses the image slicing technique to cover a field of view (FOV) of $1^{\prime} \times 1^{\prime}$ in wide-field mode (WFM) resulting in a sampling of $0.2^{\prime \prime} \times 0.2^{\prime \prime}$ spaxels (equivalent to $0.016 \mathrm{kpc}$ at a distance of $16.9 \mathrm{Mpc}$ for NGC 4710). The full field is split up into 24 subfields (each $2.5^{\prime \prime} \times 60^{\prime \prime}$ in WFM) that are fed into one of the 24 integral field units (IFUs) of the instrument. In addition, MUSE covers an impressive wavelength range from $4650 \AA$ to $9300 \AA$ at a spectral resolution of $R \sim 2000$ at $4600 \AA$ and $R \sim 4000$ at $9300 \AA$.

We observed the bulge of NGC 4710 using MUSE without adaptive optics at the nominal wavelength range. The central coordinates of the observed field ( $\alpha=12 \mathrm{~h} 49 \mathrm{~m} 37.9 \mathrm{~s}, \delta=$ $+15^{\circ} 10^{\prime} 00.8^{\prime \prime}, \mathrm{J} 2000$ ) had an offset with respect to the centre of the galaxy to avoid contamination from a bright star in the FOV. This configuration allowed us to cover the inner $15^{\prime \prime}$ of the $\mathrm{X}$-shape bulge and to extend the coverage to the expected limit of the bulge at the NW side of the galaxy.

Stellar kinematic maps of NGC 4710 were previously constructed by the SAURON and ATLAS ${ }^{3 \mathrm{D}}$ surveys. Although these 


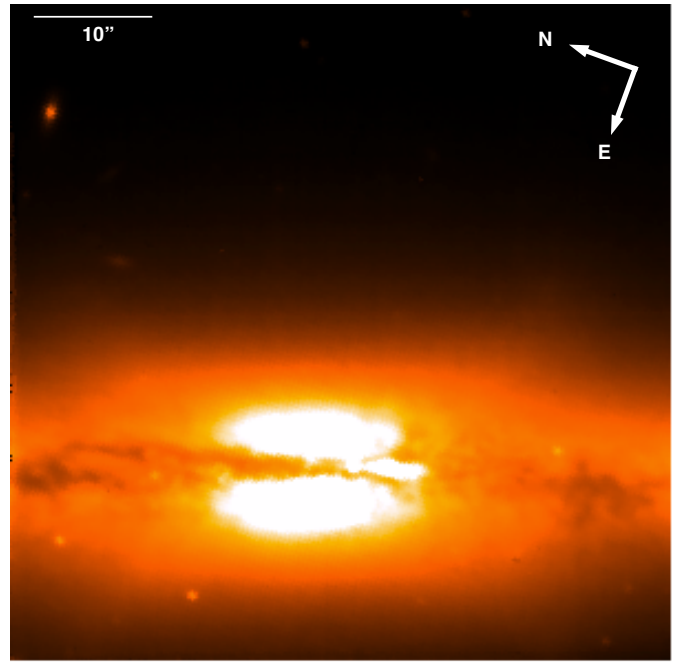

Fig. 1. Reconstructed image of the MUSE cube resulting from the coaddition of four individual exposures mapping the bulge of NGC 4710 The bar in the upper left panel indicates a scale of $10^{\prime \prime}$.

surveys provided a large set of homogeneously analysed galaxies, the larger FOV and spatial resolution of MUSE allows for a superior, detailed analysis of the kinematical signatures present in a single galaxy. In this case, the MUSE dataset is superior both with respect to the spatial coverage that allows us to trace the BP bulge to the external regions of NGC 4710 and a spatial resolution of $0.2^{\prime \prime}$ in the inner regions.

A total of four exposures of 600s were obtained, each of which was followed by a sky exposure obtained in an offset field ( $\left.\alpha=12 \mathrm{~h} 49 \mathrm{~m} 48.5 \mathrm{~s}, \delta=+15^{\circ} 06^{\prime} 32.1^{\prime \prime}, \mathrm{J} 2000\right)$. We adopted an exposure time of $180 \mathrm{~s}$ for the sky exposures. We iterated between two rotator angle positions of $-28.8^{\circ}$ and $90^{\circ}-28.8^{\circ}=$ $61.2^{\circ}$ between each integration. Figure 1 shows the reconstructed image of the MUSE cube; the BP bulge of NGC 4710 is clearly visible.

The data were reduced using the MUSE pipeline (v1.0) and using the legacy static calibrations provided by ESO for science observed during science verification runs. The reduction process of the individual scientific exposures was performed executing the specifically designed MUSE pipeline recipes. The final datacube analysed here corresponds to the combined outcome of the four individual observations. Sky subtraction was performed using our dedicated sky observations with the subtract-model method and a sky fraction of $85 \%$ to account for possible contamination from the foreground and faint halo of NGC 4710, which could still be present in our offset field. Astrometric calibrations were calculated by the pipeline using the static calibration database. We performed telluric correction and flux normalisation via observations of standard star GD153 observed by the MUSE-SV team immediately before the start of our observations. For the present study, we only focused on the spectral region between $4750-6100 \AA$ and for this reason the pipeline reduction procedure was restricted to this region. This limited wavelength coverage facilitated the complicated sky subtraction and telluric correction arising when reducing the complete MUSE wavelength region.

Once the final cube was constructed, the next step was to use the Voronoi binning method of Cappellari \& Copin (2003) to spatially bin the cube to maximise the spatial resolution while providing a minimum signal-to-noise $(\mathrm{S} / \mathrm{N})$ ratio of 50 in each spatial bin. Setting this $\mathrm{S} / \mathrm{N}$ limit allows for a proper analysis of
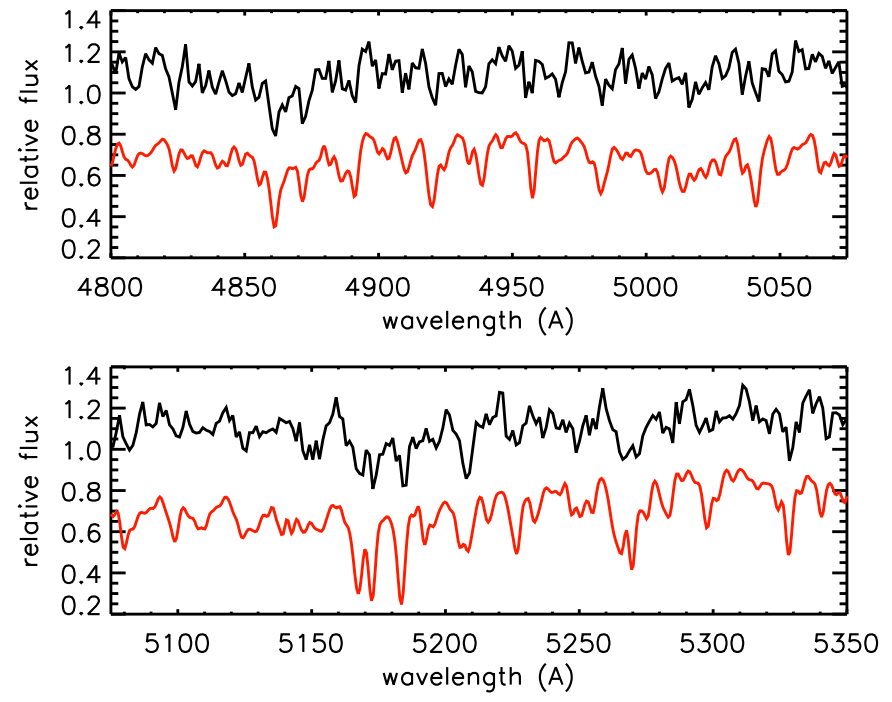

Fig. 2. Example spectrum of NGC 4710 corresponding to one bin resulting from the Voronoi binning with signal-to-noise 50 (black line). The spectral region shown corresponds to the spectral region used on the penalised pixel fitting. A template spectrum from the Vazdekis et al. (2010) library is also included as a reference (red line).

the stellar kinematics of the bulge of NGC 4710 to be carried with confidence in our entire FOV. Figure 2 shows an example of the spectra corresponding to one spatial bin of the resulting MUSE cube.

We carried out the kinematic analysis of the resulting spectra in each spatial bin using the penalised pixel fitting (pPXF) routines in IDL developed by Cappellari \& Emsellem (2004). We used the Single Stellar Population library of Vazdekis et al. (2010) as reference. This template library has the same resolution as the analysed spectral range covered by MUSE (FWHM $2.3 \AA$ ) and thus the convolution of the template spectra was not necessary. Gas emission lines are masked-out from the fit by pPXF which then evaluates the galaxy stellar kinematics by fitting the templates to the observed spectrum in pixel space using a maximum penalised likelihood method. The first four terms of the Gauss-Hermite series are then extracted from fitting the line-of-sight velocity distribution (LOSVD), providing the LOS mean velocity $V$, the velocity dispersion $\sigma$, and the next two Gauss-Hermite coefficients h3 and h4. The LOS mean velocity and velocity dispersion $(\sigma)$ are used to construct rotation maps that can be directly compared to those constructed in the Milky Way bulge based on the GIBS data (Zoccali et al. 2014). We do not include the h3 and h4 moments in our analysis because MUSE has an instrumental line spread function (LSF) which is strongly not-Gaussian. Moreover, the LSF varies on a spaxel-by-spaxel basis and also has some wavelength dependence. Therefore, the h3 and h4 measurements do not only contain the information on the galaxy's orbital distribution, but are also contaminated by the MUSE instrumental LSF. This contamination is negligible in the hot regions, dominated by velocity dispersion (e.g. $\sigma>90 \mathrm{~km} \mathrm{~s}^{-1}$ ), but becomes more important in those regions characterised by $\sigma$ similar to the instrumental $\sigma$ of MUSE (e.g. outer regions, where $\sigma \sim 50 \mathrm{~km} \mathrm{~s}^{-1}$ ). It is however important to keep h3 and h4 in the fit performed by pPXF because otherwise all the instrumental effects contaminate our measurements of $V$ and $\sigma$. The typical errors in our $V$ and $\sigma$ measurements are 6 and $8 \mathrm{~km} \mathrm{~s}^{-1}$, respectively. 

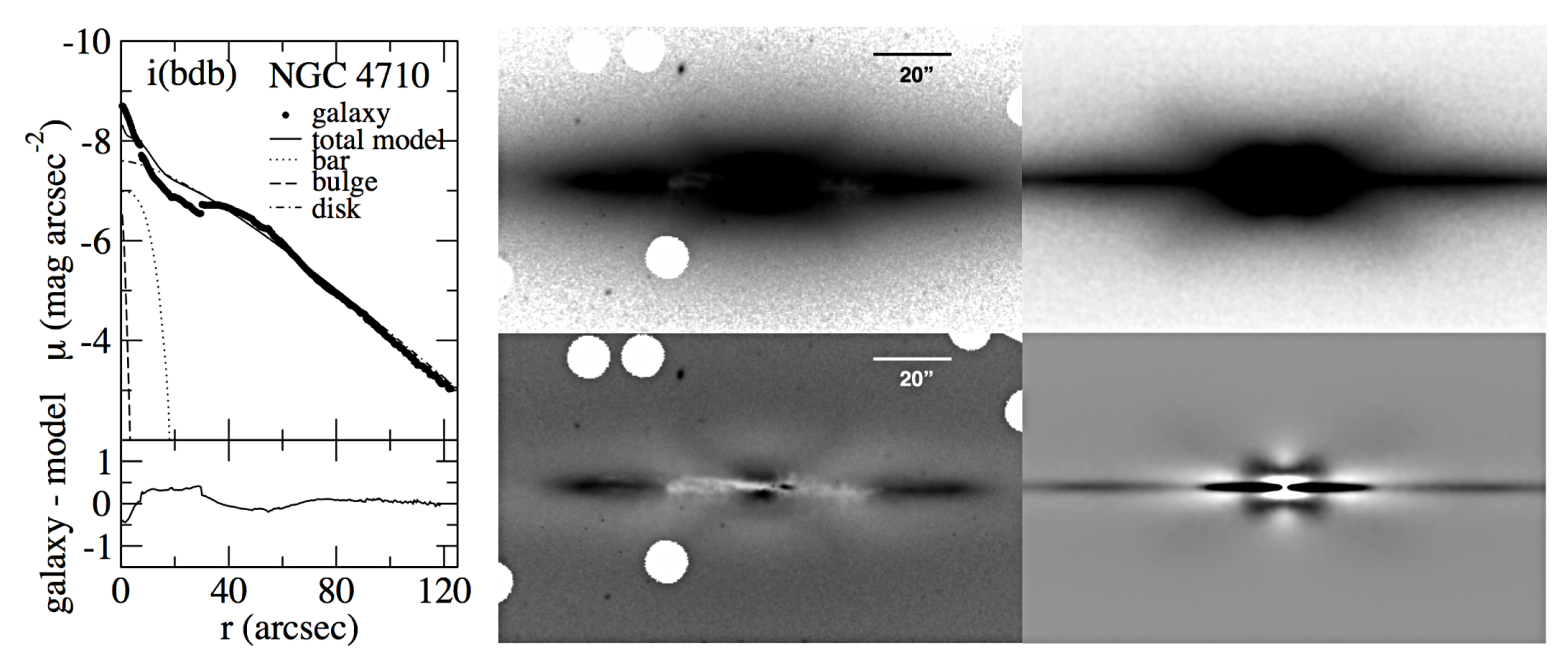

Fig. 3. Left panel: intensity map of our NGC 4710 MUSE field. Middle and right panels: LOS velocity and velocity dispersion maps, respectively. Each map has the corresponding isophotes overplotted, spaced by one magnitude. The table with LOS velocities is available for download at http://www .oagonzalez.net/data.html

\section{Structural components in NGC $\mathbf{4 7 1 0}$}

In order to correctly interpret the rotation and velocity dispersion maps, it is crucial to understand the structural composition of the sampled region of NGC 4710. With this approach, we know which component (or components) dominates the spectra in each region. We thus refer to the analysis of Gadotti (2012) where a decomposition of NGC 4710 was performed using BUDDA (de Souza et al. 2004; Gadotti 2008) to decompose the $i$-band image of NGC 4710 obtained from the Sloan Digital Sky Survey (SDSS) into different structural components.

Figure 3 shows the best-fit light profile for NGC 4710 from Gadotti (2012). The best model for NGC 4710 includes a vertically extended bar structure. On the other hand, the best-fit model includes a small structure with a Sérsic index of 0.7 that contributes only with a very minor fraction, $0.1 \%$, to the total luminosity of the galaxy. Its near-exponential light profile suggests an origin that is connected to the main exponential disc, so it is likely to be a structural component built from disc material. Figure 3 shows the residual image after the BUDDA model subtraction. The clear X-shape obtained in the residual image strongly corroborates that the bulge of NGC 4710 belongs to the family of BP bulges.

In addition, the residual image in Fig. 3 also shows clearly a few substructures that may be associated with the bar. Firstly, as seen in Gadotti (2012), beyond the dust lane, at each side of the centre, a narrow excess of light indicates the presence of a density enhancement in the disc that could be the bar ansae, spiral arms, or a ring. These structures appear to be common in edge-on galaxies with BP bulges (e.g. Bureau et al. 2006), and in simulations of barred galaxies when viewed at an edge-on projection (e.g. Laurikainen \& Salo 2016).

Secondly, in the central $\sim 5^{\prime \prime}$ of the residual image one notices a small structure that sticks out prominently from the disc plane (although it is still significantly less extended that the BP bulge in the vertical direction). This structure has a pronounced boxy shape, which hints at it being associated with the largescale BP. Although the model fitted has a component that accounts for the BP, this model is designed to account for its average light distribution, so deviations from the average stand out in the residual image. That would be the case if the central light distribution exceeds what is expected in the global BP model, hence producing the observed residual image. To test whether this central structure is just part of the BP, similar to the X-shape residual component, we performed a structural analysis using BUDDA on a snapshot of the galaxy simulation of Cole et al. (2014); the same type of analysis that was performed with the SDSS image of NGC 4710. The simulation contains only a disc and bar (plus BP) components, and the snapshot was convolved with a circular Gaussian function to mimic PSF effects. The Gaussian FWHM was chosen to reproduce the same relative PSF as in the SDSS image of NGC 4710. As found for NGC 4710, the best BUDDA model essentially contains only a disc and a bar component. The residual image derived from this fit is shown on the lower right of Fig. 3. One clearly sees both the large-scale $\mathrm{X}$-shape residual component and a central smaller structure that is very similar to that found in the residual image of NGC 4710. However, in this case, the central structure is most likely associated with the BP, as indicated by the conspicuous X-shape it has. We conjecture that the corresponding X-shape of the central structure is not entirely discernible in the residual image of NGC 4710 because of a combination of projection effects and spatial resolution of the SDSS image. If the galaxy is not seen at a perfect side-on projection the smaller, innermost $\mathrm{X}$-shape structure may appear only as boxy.

Thus, in the case of NGC 4710, the structural analysis indicates that our kinematic study traces the dynamical signatures dominated by the disc and bar, in the regions near the plane of the galaxy, and that of the associated BP bulge at increasing height.

\section{Kinematic maps of NGC $\mathbf{4 7 1 0}$}

We have used the resulting kinematics derived from the absorption-line fitting with pPXF to produce kinematic maps of NGC 4710. Figure 4 shows the intensity map obtained from collapsing our datacube across the spectral dimension; the figure also shows the maps of LOS velocity and velocity dispersion that are produced after correcting for the corresponding redshift of NGC $4710\left(V=1174 \mathrm{~km} \mathrm{~s}^{-1}\right)$. The isophotes obtained from the reconstructed image are overplotted in each map and are spaced by one magnitude intervals. We find the kinematic PA of the galaxy to be $24.8^{\circ} \pm 3.1^{\circ}$, and we obtained this PA using the method described in Appendix C of Krajnović et al. (2006) 

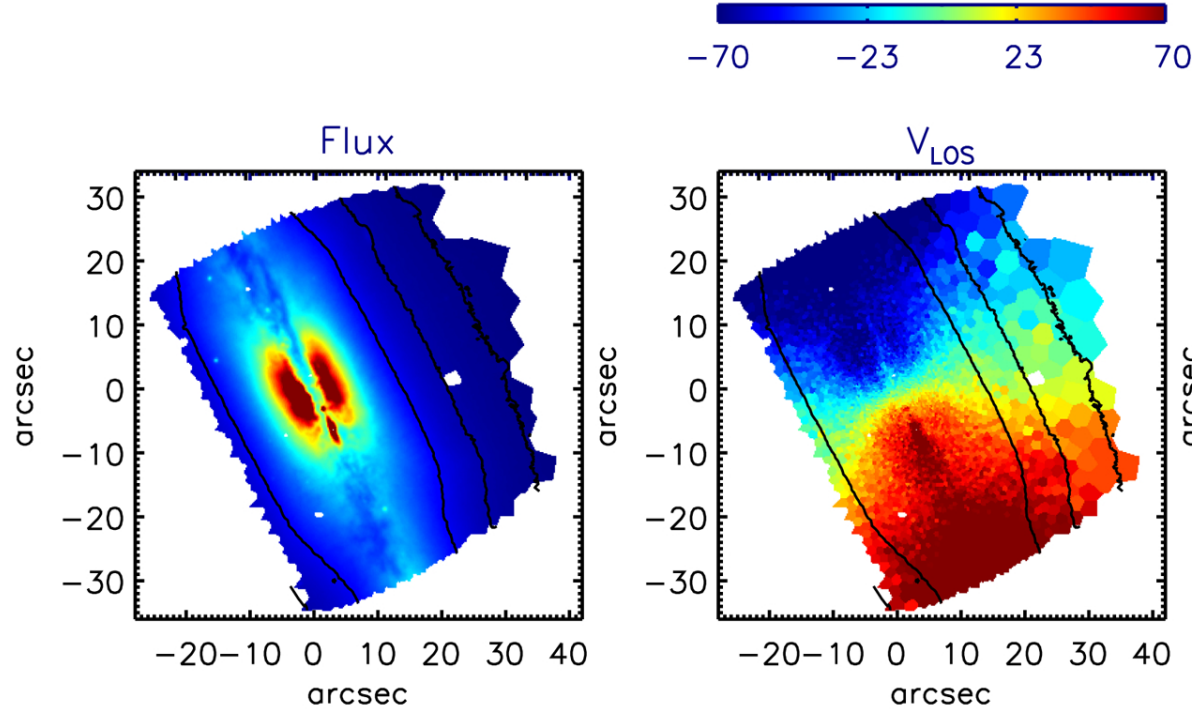

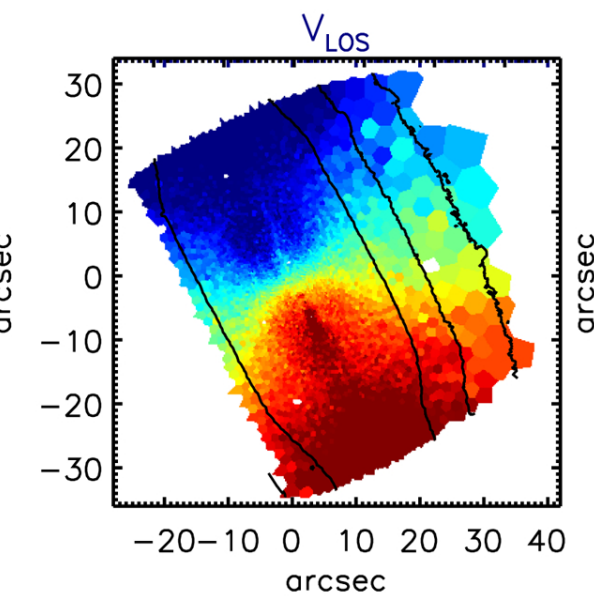

$50 \quad 70 \quad 90 \quad 110$

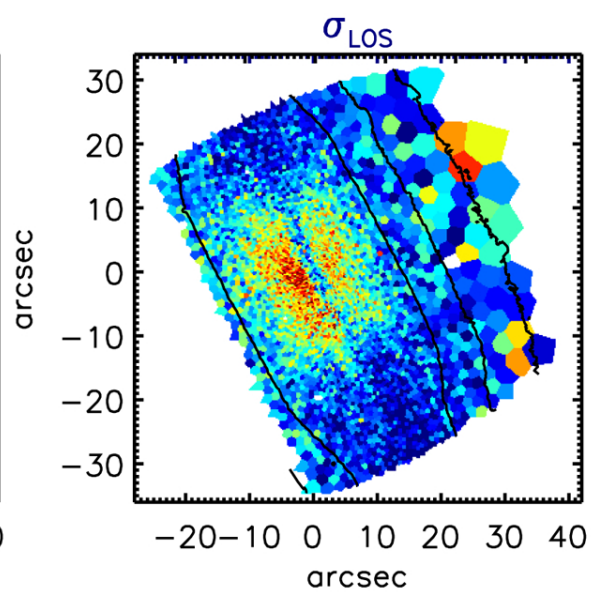

Fig. 4. Left panel: intensity map of our NGC 4710 MUSE field. Middle and right panels: velocity dispersion and LOS velocity maps, respectively. Each map has the corresponding isophotes overplotted, spaced by one magnitude. The table with LOS velocities is available for download at http://oagonzalez.net/data.html

and implemented in the IDL routine FIT_KINEMATIC_PA by M. Capellari.

The LOS velocity map shows the signatures of cylindrical rotation, as expected for a BP bulge, which is consistent with the photometric analysis of NGC 4710. In theory, a bulge rotating perfectly cylindrically would have a mean LOS velocity, at a given distance from the centre, which is independent of its height above the plane (Saha \& Gerhard 2013). A very useful criteria to quantify the deviations from pure cylindrical rotation was provided by Eq. (2) of Saha \& Gerhard (2013) as follows:

$\delta_{\mathrm{CL}}\left(z, X_{j}\right)=\frac{\int_{0}^{X_{j}} \Sigma_{\mathrm{los}}(x, z) V_{\mathrm{los}}\left(x, z_{i}\right) x^{2} \mathrm{~d} x}{V_{\mathrm{los}}\left(R_{\mathrm{b}, 1 / 2}, z \simeq 0\right) \int_{0}^{X_{j}} \Sigma_{\mathrm{los}}(x, z) x^{2} \mathrm{~d} x}$,

where $\Sigma_{\text {los }}(x, z)$ is the surface density, $V_{\text {los }}(x, z)$ is the LOS velocity, $X_{j}$ is the projected distance from the minor axis for a slit at a height $z=z_{i}$, and $V_{\mathrm{los}}\left(R_{\mathrm{b}, 1 / 2}, z \simeq 0\right)$ is the velocity at the bulge half-mass radius close to the disc mid-plane. We then fit a straight line to the $\left(\delta_{\mathrm{CL}}, z\right)$ curve and derive the slope of this relation $m_{\mathrm{CL}}$. Saha \& Gerhard (2013) then defines the degree of cylindrical rotation in the BP bulge as

$\delta_{\mathrm{CL}}^{n}\left(X_{j}\right)=1+m_{\mathrm{CL}}$,

so that a value of $\delta_{\mathrm{CL}}^{n}=1$ corresponds to perfect cylindrical rotation while values of $\delta_{\mathrm{CL}}^{n}<0.75$ correspond to non-cylindrical rotation.

We applied this equation to our NGC 4710 dataset measuring the surface density and LOS velocity across fixed heights from the plane. Within the half-mass bulge radius of NGC 4710, which corresponds to $R_{\mathrm{b}, 1 / 2}=1.35 R_{\mathrm{e}}=10^{\prime \prime}$ (where $R_{\mathrm{e}}=7.4^{\prime \prime}$, Gadotti 2012), we obtain a value of $\delta_{\mathrm{CL}}^{n}=0.9$. The slight deviation from cylindrical rotation seen in NGC 4710 could be a consequence of either the presence of a rotating classical bulge or the effect of the bar, and associated BP, orientation angle. Following the structural decomposition of NGC 4710 of Gadotti (2012), the presence of a classical bulge in this galaxy can be safely discarded and thus the deviation from cylindrical rotation is likely to be the result of the position angle of the bar. The impact of the bar viewing angle in the measured rotation of the associated BP bulge was discussed in Combes et al. (1990) and Athanassoula \& Misiriotis (2002) and was recently investigated in detail by Iannuzzi \& Athanassoula (2015); these results are consistent with the very small deviation from cylindrical rotation we see in the BP bulge of NGC 4710.

The general behaviour of the maps is in good agreement with the expectations from $N$-body models presented in Iannuzzi \& Athanassoula (2015) for edge-on galaxies hosting BP bulges. The velocity dispersion increases noticeably towards the inner regions of the BP bulge. We note that the velocity dispersion map shows an asymmetric profile with a higher velocity dispersion towards the north-east side of the galaxy. This most likely is the consequence of the disc of NGC 4710 not being perfectly edge-on.

Additionally, we note a very bright spot-like region located in the disc plane of NGC 4710, which is clearly seen in Fig. 4 at $(X, Y)=\left(4^{\prime \prime},-6^{\prime \prime}\right)$. This brighter region could be the result of a dust-free patch in the disc that might allow us to see deeper through the disc up to the inner regions of the bulge. However, the radial velocity map shows a peak in mean velocity for this region, which can be interpreted as a signature of this region being located in the near side of the disc. Furthermore, the spectra show an increase of emission lines in this region, suggesting that this is a gas-rich region in the disc.

\section{Stellar populations in the bulge of NGC $\mathbf{4 7 1 0}$}

The stellar populations of bulges and their connection with the processes involved in the bulge formation remains a matter of debate. The different types of bulges lead to expectations on their stellar population content based on their formation scenario. However, the observations of bulges, including that of the Milky Way, show a variety of stellar ages and metallicities that do not always match the expectations of their classification based on their morphology and/or kinematics. Different models and bulge formation theories have explained this lack of a one-to-one relation between the morphological classification of bulges and their observed stellar population properties based on the possibility of a given bulge having a mixture of components, each with its own formation history. Similarly, a variety of properties can be 
expected for any given component, depending on the characteristics of the processes involved. For example, a wide range of stellar ages can be found even among BP bulges. Stellar bars in massive galaxies, such as those found in NGC 4710 and in the Milky Way, are expected to have already formed at redshift $\sim 1$ (Sheth et al. 2012) or earlier (Simmons et al. 2014; Gadotti et al. 2015). The buckling instability of bars, and thus the formation of the BP bulge, can take place on very short timescales ( $\sim 1 \mathrm{Gyr}$, Athanassoula 2008), opening the possibility of having mostly old stellar ages in BP structures. On the other hand, a fraction of younger stars can still be present in BP bulges, provided that star formation has continued in the disc, within the radius at which the bar ends so that these younger stars can be captured by the bar potential. As a consequence, this young population of stars would be expected to be predominantly near the plane (Ness et al. 2014; Dékány et al. 2015).

In this context, NGC 4710, which hosts only a pure BP bulge, is an excellent laboratory to investigate the stellar population properties that can be found in such structures directly. With this aim, we used the full-spectrum fitting capabilities of $\mathrm{pPXF}^{2}$ (Cappellari \& Emsellem 2004) to investigate the distribution of the mass fraction in the bulge of NGC 4710 in terms of age and metallicity. A grid of 156 MILES model spectra from Vazdekis et al. (2010; 26 ages, $6[\mathrm{M} / \mathrm{H}])$ was used in pPXF to obtain a best-fit spectrum for five representative regions of the bulge. pPXF searches for an optimal solution that is based on the weights applied to each template spectrum in the age-metallicity grid using a regularisation parameter. The regularisation constrains the solution in such a way that the weights assigned to neighbouring age and metallicity templates changes smoothly while being consistent with the observed spectrum. The final representative stellar population of the observed integrated spectrum is then obtained by calculating a weighted average of the metallicities and ages of the grid using the weights calculated by $\mathrm{pPXF}$. We found that these average values have a negligible sensitivity to the selected regularisation parameter. Thus, we used the default regularisation parameter of 250 , which corresponds to a parametrisation error that is consistent with the typical weights assigned to the templates.

We applied the spectral fitting procedure to the integrated spectra of four fields located along the minor axis of the BP bulge of NGC 4710, specifically at heights from the plane of $0.3,0.7$, 1.1 , and $1.5 \mathrm{kpc}$ and derived its dominant age and metallicity. All four fields appear to be consistently dominated by a relatively old population $(>5$ Gyr) with a mean age of $\sim 9$ Gyr. On the other hand, metallicity decreases as a function of height from the plane of the galaxy. Figure 5 shows the metallicity of the bulge of NGC 4710 in each field compared to the value of the Milky Way bulge at an equivalent height from the plane. As a reference for the minor axis of the Milky Way bulge, we use the photometric metallicity maps of Gonzalez et al. (2013) and the spectroscopic measurements from Zoccali et al. (2008) and Johnson et al. (2013).

The stellar population analysis of the BP bulge of NGC 4710, shown in Fig. 5, suggests a scenario where NGC 4710 has relatively old stellar ages and a vertical metallicity gradient comparable to that in the bulge of the Milky Way. The metallicity gradient in the bulge of NGC 4710 is an important observational confirmation of vertical gradients reproduced in the simulations

\footnotetext{
2 See http://www-astro.physics.ox.ac.uk/ mxc/software/ for a detailed description of pPXF and the implementation of regularisation when assigning weights to the library templates when performing the penalised fitting procedure.
}
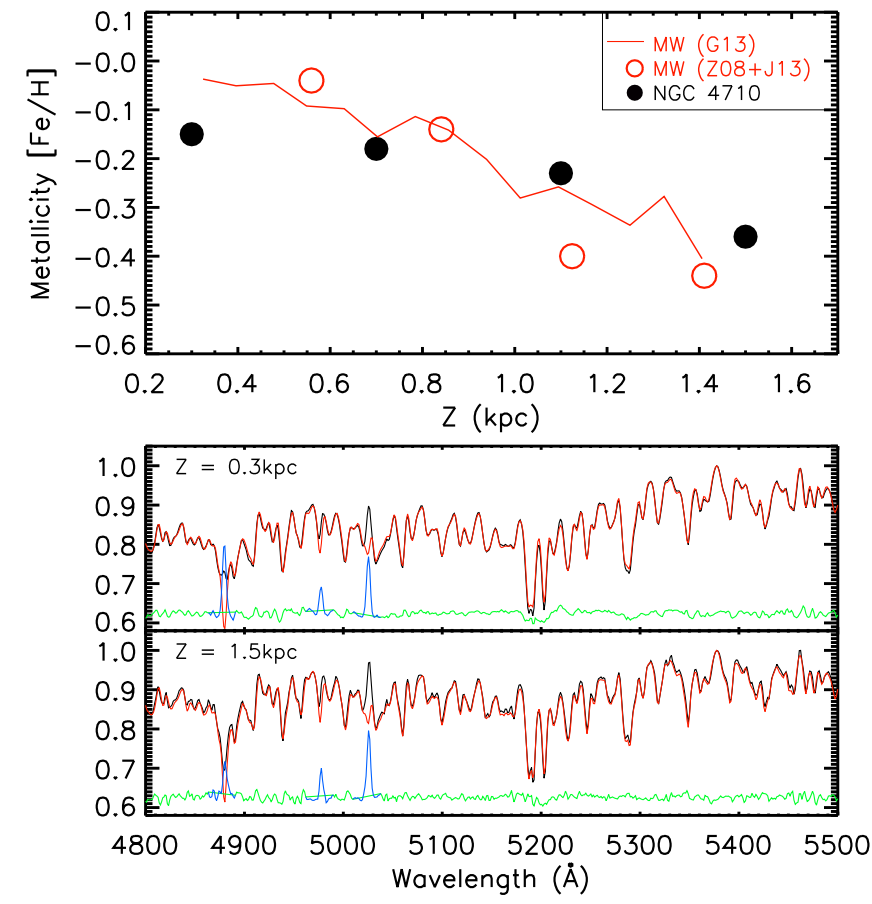

Fig. 5. Stellar population solution of pPXF from the full spectral fitting procedure in four regions along the minor axis of NGC 4710. The upper panel shows the mean metallicity of the bulge of NGC 4710 at different distances from the plane $(Z)$ indicated with black filled circles. Mean metallicity measurements for the bulge of the Milky Way are overplotted. The photometric measurements from Gonzalez et al. (2013) are shown with red solid lines, while the spectroscopic measurements from Zoccali et al. (2008) and Johnson et al. (2013) are indicated with empty red circles. The two lower panels show the observed (black solid line) and best-fit spectrum (red solid line) of the inner and outermost regions of the bulge of NGC 4710, the masked emission line regions (blue solid lines), and the fitting residuals (green).

of pure BP bulges seen edge-on (Bekki \& Tsujimoto 2011; Martinez-Valpuesta \& Gerhard 2013). The exact value of the gradient must be taken with caution as the innermost field is probably affected by a significant contribution of disc stars. However, we note that the scale height of the disc component in the BUDDA model light decomposition of NGC 4710 is $\sim 0.8 \mathrm{kpc}\left(10.3^{\prime \prime}\right.$ at $\left.16.9 \mathrm{Mpc}\right)$. Therefore, the disc contamination is expected to be negligible at the heights of the two fields at 1.1 and $1.6 \mathrm{kpc}$ from the plane where a variation in metallicity of $0.14 \mathrm{dex}$ is observed. Detailed maps of $[\mathrm{Fe} / \mathrm{H}], \alpha$-element, and stellar ages based on the measurement of line-strength indices, are the subject of a dedicated study (Gonzalez et al., in prep.).

\section{Comparison of the bulge of NGC 4710 and the Milky Way}

The advent of spectroscopic and photometric surveys covering a large area of the Milky Way bulge are revealing properties that were previously inaccessible to us. One of the most recent observational findings has been that the Galactic bulge hosts a BP or $\mathrm{X}$-shaped structure. Such components are often found in external galaxies with a suitable orientation. Usually, a single deep image might already provide an answer to whether its bulge hosts this kind of structure. However, characterising the Milky Way bulge structure requires an entirely different approach based on the morphological reconstruction of an enormous amount of data where the distance estimation is obtained from the magnitudes 
of red clump giant stars (Zoccali 2010; McWilliam et al. 2010; McWilliam \& Zoccali 2010; Nataf et al. 2010; Saito et al. 2011; Wegg \& Gerhard 2013). Such a detailed star-by-star reconstruction of the properties of the bulge are only possible in the Milky Way. Understanding bulges in external galaxies requires combining the interpretation of properties obtained from different techniques, such as image decomposition and spatially resolved kinematics. Thus, providing a link between techniques used in external galaxies and in the Milky Way is of great importance.

Zoccali et al. (2014) derived radial velocities for a sample of 6390 bulge red-clump stars from the GIBS survey. They constructed rotation curves at four different latitudes $\left(b=-2^{\circ},-4^{\circ}\right.$, $-6^{\circ}$, and $\left.-8^{\circ}\right)$ and interpolated between their fields to obtain the first rotation and velocity dispersion maps of the Milky Way bulge. We can use our maps of NGC 4710, where the entire kinematics could be measured directly, to compare to those of Zoccali et al. (2014). We thus transformed the radial velocity and velocity dispersion maps of NGC 4710 into a reference frame that is comparable to that of the Galactic bulge as seen from the Sun. We first transformed the photometric centre of NGC 4710 ( $\alpha=12 \mathrm{~h} 49 \mathrm{~m} 38.8 \mathrm{~s}, \delta=+15^{\circ} 09^{\prime} 56.9^{\prime \prime}$; Gadotti 2012) to pixels using the astrometrical solution of our MUSE reconstructed image. The centre of NGC 4710 is found in our image at pixel positions $x=141, y=189$. We used these pixel values as the new reference centre and further applied a rotation of $64^{\circ}$ to obtain a new reference frame $\left(x^{\prime}, y^{\prime}\right)$ centred on NGC 4710, where the position angle of the major axis of the galaxy is zero. On the other hand, the pixel scale of MUSE of $0.2^{\prime \prime} / \mathrm{pix}$ indicates that each pixel in our map would be equivalent to $0.016 \mathrm{kpc}$ at the distance of NGC 4710 (16.9 Mpc). In the Milky Way, the bulge is located at approximately $8 \mathrm{kpc}$ from the Sun, such that an angular size of 1 deg corresponds to $0.139 \mathrm{kpc}$. Thus, if the bulge of NGC 4710 were located at $8 \mathrm{kpc}$ from the Sun, each resolving element of our maps would have 0.016/0.139 $=0.11 \mathrm{deg} / \mathrm{pix}$. Applying this new scale, we constructed new maps for NGC 4710 in a reference frame that can be compared directly to the Galactic coordinate system of the Milky Way.

We also included in this comparison the corresponding kinematic maps for the BP bulge of the simulation of Cole et al. (2014; see Fig. 1 and Sect. 3). We applied a scaling factor of 1.2 to the model coordinates to scale its bar size of $R_{\mathrm{bar}}=2.9 \mathrm{kpc}$ to the bar size of the Milky Way ${ }^{3}$, as described in Ness et al. (2014). For comparison purposes, the corresponding radial velocities and velocity dispersions have also been normalised so that the maximum radial velocity of all the bulges is comparable to that of NGC 4710 by applying a scaling factor of 0.48 to the velocities of the model and of 0.77 to the velocities measured for the Milky Way by Zoccali et al. (2014). Such spatial and velocity scaling transformations changes the reference systems but have no effect on the rotational patterns we aim to investigate.

This simple exercise allows us to visually compare the kinematical patterns of NGC 4710 with those of the Milky Way obtained via the different Milky Way surveys. The position angle of the bar of NGC 4710 with respect to our LOS must be much closer to a side-on projection than for the case of the Milky Way, as suggested by the direct detection of the pronounced X-shaped bulge. This gives us the chance to investigate the effects of the bar orientation angle in the kinematic maps of the Milky Way bulge from Zoccali et al. (2014).

\footnotetext{
We note that the length of the Milky Way bar has been measured to be $4.4 \mathrm{kpc}$ in the Galactic plane. However, since here we are scaling the inner parts of the bar that form the BP bulge, we use the length of $3.5 \mathrm{kpc}$ measured at larger distances from the Galactic plane (Wegg et al. 2015).
}

We can immediately see a similarity between the LOS velocity map of the bulge of NGC 4710 and that of the Milky Way in Fig. 6. This is particularly true when considering that the apparent higher smoothness of the velocity dispersion map for the Milky Way, presented in Zoccali+14, with respect to that NGC 4710 is a natural consequence of the different adopted techniques. For the Milky Way case, the map was obtained interpolating between the GIBS fields grid. In addition, it can be seen that the central peak in velocity dispersion that is found in the central region of the Milky Way bulge map is not seen with the same concentration and vertical elongation in the maps of NGC 4710. As already pointed out by Zoccali+14, while the presence of the central sigma peak is strongly supported by accurate velocity measurements of a large and statistically robust sample (450 individual stars), the extension of the peak is however poorly constrained because of the presence of only two fields in that region. Similar changes in the central velocity dispersion profiles are among the features identified in the LOS kinematics maps of Iannuzzi \& Athanassoula (2015) and they are attributed to the effect of bar position angle. We see a similar change in the velocity dispersion maps constructed using different bar orientation angles in the simulation. Figure 6 shows how the $\sigma$ map of the simulated BP bulge becomes less vertically elongated when seen at (or close to) side-on projection, which is the case of NGC 4710. However, in this case the elongated central feature is not as pronounced as seen in the velocity dispersion map of the Milky Way bulge.

We now look at the 1D LOS velocity and velocity dispersion profiles. In particular, we investigate their variations as a function of height to compare the velocity dispersion of the innermost fields of the Milky Way and NGC 4710 in a more quantitative way. To make this comparison, we scaled the $\left(x^{\prime}, y^{\prime}\right)$ spatial coordinates of NGC 4710 by a factor 0.88 corresponding to the ratio between the bar sizes of the Milky Way $R_{\mathrm{bar}}=3.5 \mathrm{kpc}$ and NGC 4710 of $R_{\text {bar }}=3.9 \mathrm{kpc}$. With these transformations, we have all three datasets spatially scaled to the bulge of the Milky Way. The velocity profiles for NGC 4710 are then constructed at the four Milky Way heights presented in Zoccali et al. (2014), i.e. $Z_{\mathrm{MW}}=0.28,0.56,0.83,1.12 \mathrm{kpc}$, which they used to interpolate the velocity maps. The $1 \mathrm{D}$ rotation and velocity dispersion profiles shown in Fig. 7 for the corresponding heights in NGC 4710 have a remarkable similarity to those of the Milky Way bulge and the differences seen between the inner regions of the $\sigma$ maps of the Milky Way and NGC 4710 are not as evident in the $1 \mathrm{D}$ curves. This suggests that the vertical elongation observed in central region of the $\sigma$ map of the Milky Way bulge might not be real, as suggested by Zoccali et al. (2014). Instead this elongation it is an artefact from the plane interpolation method when including the $\sigma$ peak observed at $b=-2$. In this case, the $\sigma$ peak in the Milky Way bulge would be limited to Galactic latitudes $|b|<2(\sim 0.28 \mathrm{kpc})$. This is also in good agreement with the conclusions of Valenti et al. (2016), where the high stellar density peak of the central Milky Way bulge closely follows a more axisymmetric $\sigma$ peak than that presented in the GIBS velocity dispersion maps.

It is clearly seen in Fig. 7 that the radial velocity profiles become steeper towards the galactic plane. This same effect was seen in the Milky Way bulge using the BRAVA survey (Howard et al. 2009) and further confirmed with the inclusion of the rotation profile at $b=-2^{\circ}$ by the GIBS survey (Zoccali et al. 2014). The simulated BP bulge shows the same behaviour as in the Milky Way and NGC 4710. Combes et al. (1990) and Athanassoula \& Misiriotis (2002) among others have suggested that such a rotation pattern would be expected for a BP bulge 

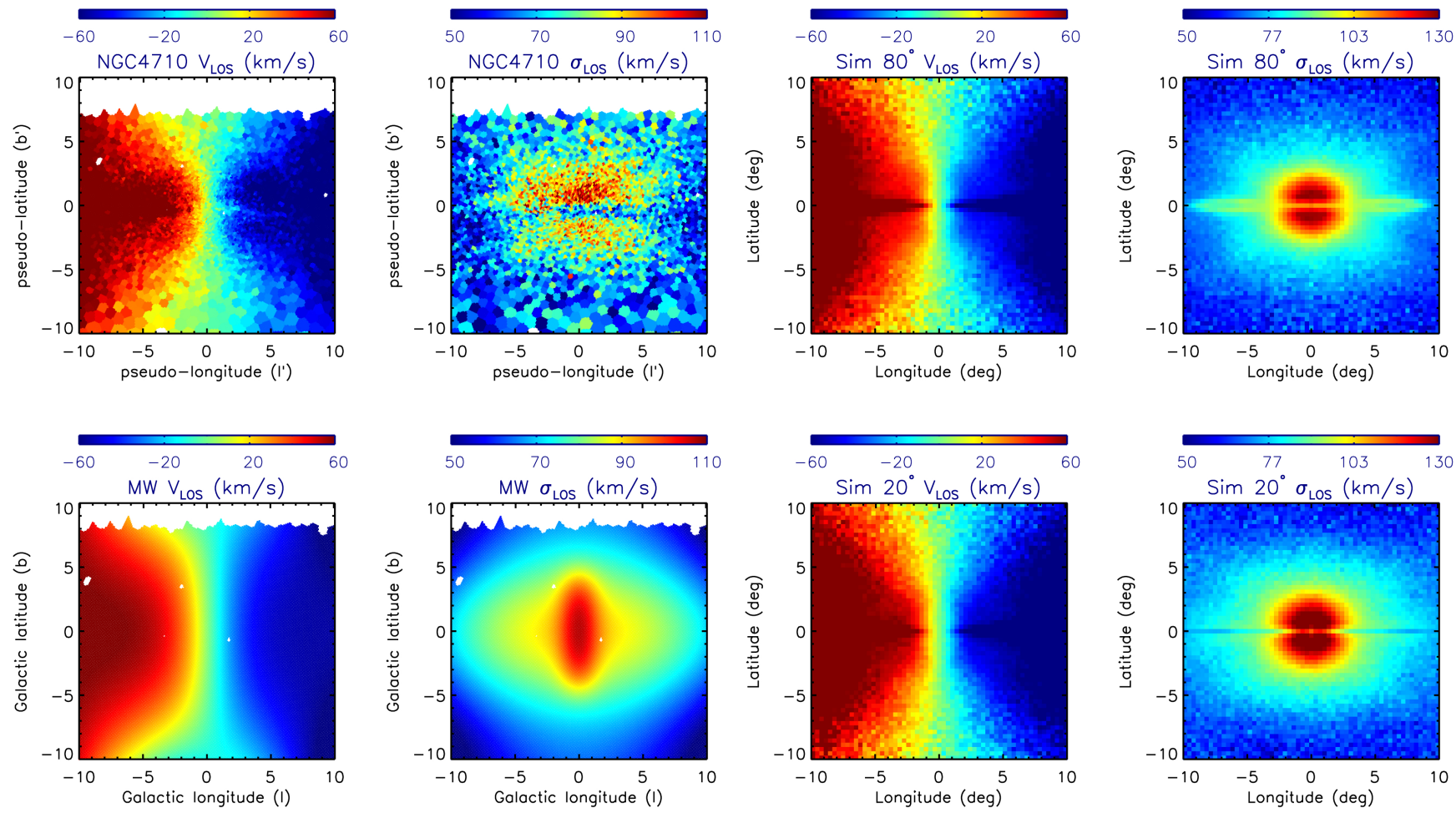

Fig. 6. Two upper left panels: LOS velocity and velocity dispersion maps of NGC 4710 projected and oriented as the Milky Way bulge is seen from the Sun. Two lower left panels: corresponding LOS velocity and velocity dispersion maps for the Milky Way bulge constructed evaluating each spatial resolution element of our NGC 4710 maps in Eqs. (1) and (2) in Zoccali et al. (2014). Right side panels: corresponding velocity maps for the simulation of Cole et al. (2014) for bar orientation angles of $80^{\circ}$ (upper panels) and $20^{\circ}$ (lower panels).

when the bar has a non-zero position angle with respect to the Sun-Galactic centre LOS and should not be immediately interpreted as evidence for the need of an additional component to reproduce the observed rotation curves. Indeed, the light decomposition of NGC 4710 itself and the very minor deviation from cylindrical rotation found in the previous section show no evidence for an additional bulge component besides the BP bulge. Furthermore, the pure-disc $N$-body models of bars that have been used to evaluate the rotation curves of the bulge show the same behaviour without the need to include a classical bulge component (Shen et al. 2010; Zoccali et al. 2014). Thus, the fact that the rotation curve of the bulge of NGC 4710 and the Milky Way are so similar suggests that both of them are dominated by a similar BP bulge structure. This is strongly supported by the detection of a vertical metallicity gradient along the BP bulge of NGC 4710 that is comparable to the metallicity gradient measured in the Milky Way bulge.

The existence of a spheroidal bulge component in the Milky Way in addition to the BP bulge, has been suggested based on the distance distribution of RR Lyrae (Dékány et al. 2013), the bi-modality in the metallicity distribution (Hill et al. 2011), and the different kinematics seen in metal-poor and metal-rich bulge stars (Babusiaux et al. 2010). Furthermore, Ness et al. (2014), Vásquez et al. (2013), and Rojas-Arriagada et al. (2014) found evidence for the X-shape bulge of the Milky Way to be only traced by the metal-rich bulge stars. The properties of the bulge of the Milky Way are in general investigated by selecting redclump stars from the colour-magnitude diagram at a given LOS. Metallicity and kinematics are then obtained individually from the spectra of each red-clump star and used to construct the total metallicity distribution. If the metal-poor component, which shows different kinematics (and spatial distribution), is much less significant in number compared to the dominant stars in the $\mathrm{BP}$, then the integrated light properties could be dominated by the BP component and would not be detectable in the integrated spectrum. This highlights the importance of understanding the detailed properties of the Milky Way bulge, where kinematics and chemical abundances can be investigated on a star-by-star basis, which is perhaps hidden in the integrated light of external galaxies. The origin of the apparent different properties between metal-poor and metal-rich stars in the Milky Way bulge, i.e. the presence of a classical bulge, remains to be fully understood. From our results we see that the integrated light of the bulge of NGC 4710, which is dominated by a BP component, is consistent with the dominant structure, kinematics, vertical metallicity gradient, and stellar ages of the Milky Way bulge.

\section{Summary and conclusions}

In this study we investigated the properties of the bulge of the edge-on galaxy NGC 4710 using MUSE spectra. The bulge of this galaxy shows a strong X-shape profile. A lightdecomposition study provides no evidence for the presence of a classical bulge in the inner regions of NGC 4710, showing instead that the integrated light from this galaxy is dominated by a bar and the associated BP bulge out of the plane of the disc.

We constructed the LOS velocity and velocity dispersion maps of the bulge, finding that the bulge rotates cylindrically, with a $\delta_{\mathrm{CL}}^{n}=0.9$, based on the definition from (Saha \& Gerhard 2013) where a value of $\delta_{\mathrm{CL}}^{n}=1$ corresponding to a bulge with perfect cylindrical rotation and values of $\delta_{\mathrm{CL}}^{n}<0.75$ define bulges with non-cylindrical rotation. The slight deviation from perfect cylindrical rotation is most likely due to the position angle of the bar being close to, but not perfectly, side on. The 

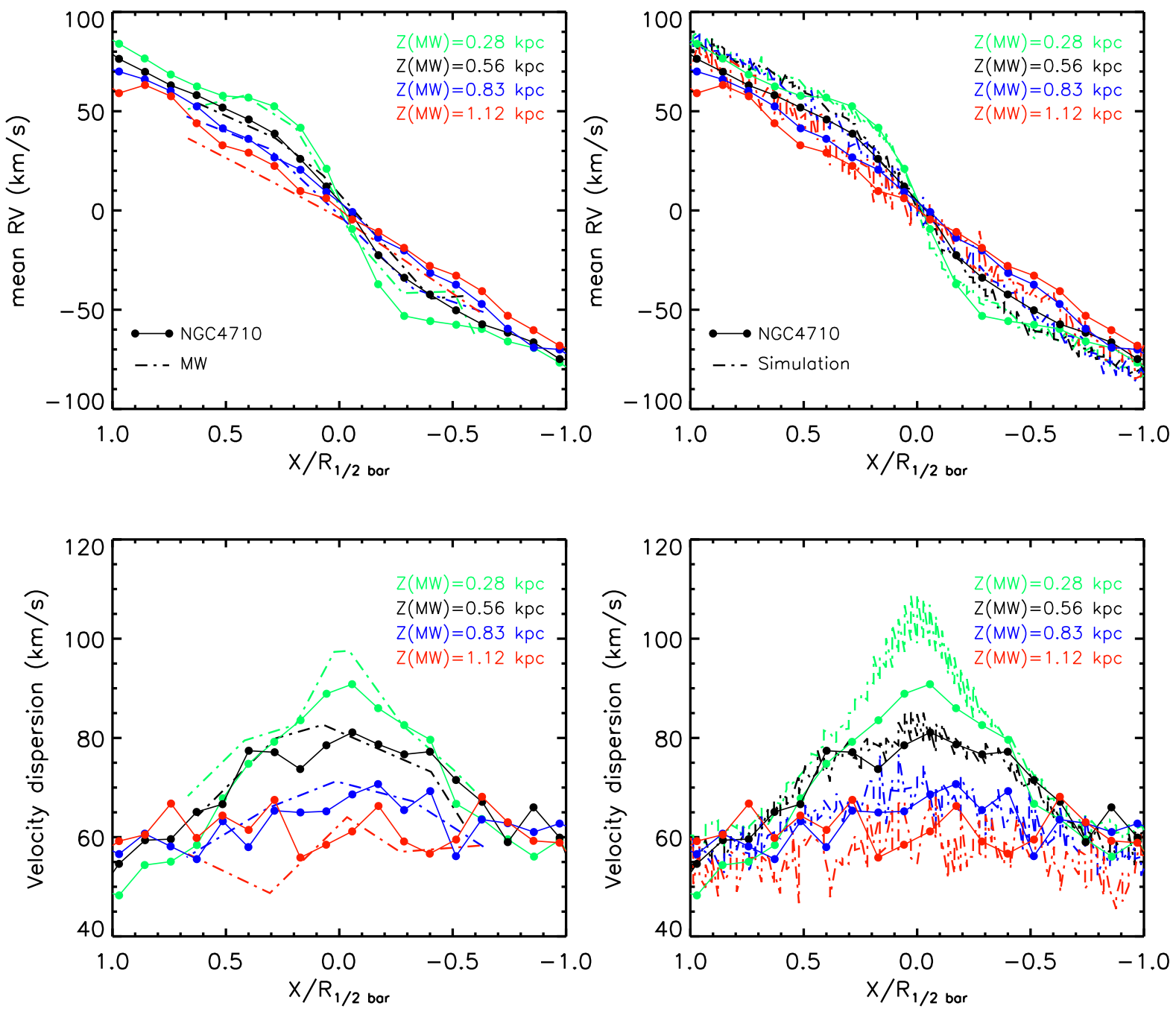

Fig. 7. Filled circles joined by solid lines show the mean LOS velocity and velocity dispersion curves of NGC 4710 at four heights from the plane $Z=0.28,0.56,0.83$, and $1.12 \mathrm{kpc}$. Dashed lines show the corresponding rotation curves of the Milky Way bulge from Zoccali et al. (2014; left panels) and of a disc galaxy simulation from Cole et al. (2014; right panels). Spatial coordinates of NGC 4710 and the simulation were scaled to match the Milky Way bulge bar scale length $\left(R_{\text {bar }}=3.5 \mathrm{kpc}\right)$. The mean velocities and velocity dispersion of NGC 4710 and the simulation were obtained in bins of $0.2 \mathrm{kpc}$ at the same scale heights of the Milky Way bulge measurements using the spatial scaling factor of 0.88 and 1.2 for NGC 4710 and the simulation, respectively. Velocities of the Milky Way bulge and the simulation were scaled to match the maximum velocity of the bulge of NGC 4710 .

velocity dispersion map shows a central peak in velocity dispersion that appears asymmetric with respect to the major axis of the galaxy, thus suggesting an edge-on view of NGC 4710 that is not perfect.

We investigated the stellar population properties of the BP bulge of NGC 4710 by performing full spectral-fitting in four fields along its the minor axis and find that the dominant population of the BP bulge is $\sim 9 \mathrm{Gyr}$ and shows no variation as a function of distance from the plane. The mean metallicity of the bulge decreases consistently at increasing heights from the plane. We measure a vertical metallicity gradient of $0.35 \mathrm{dex} / \mathrm{kpc}$ up to $1.5 \mathrm{kpc}$ from the plane of the galaxy.

We scale the maps of NGC 4710 to a system that can be directly compared with the Milky Way bulge and compare the resulting maps with those constructed by Zoccali et al. (2014) based on interpolation of the GIBS survey rotational curves. In this comparison we found that the rotation map of NGC 4710 and the rotation map of the Milky Way bulge show a remarkable similarity. Although the velocity dispersion map of the bulge of NGC 4710 appears noisier than that of the Milky Way bulge, owing to the way the maps are constructed, we see that the velocity dispersion map of the Milky Way bulge is more vertically elongated in the centre than in the velocity dispersion map of NGC 4710. We see a similar change in the velocity dispersion maps constructed using different bar orientation angles in the simulation from Cole et al. (2014). This is also in good agreement with the features identified in the LOS kinematic maps of BP bulges modelled by Iannuzzi \& Athanassoula (2015). We thus suggest that central increase in the velocity dispersion profile of the Milky Way bulge can be partially the consequence of the Milky Way bar and BP bulge viewing angle of $27^{\circ}$ with respect to the Sun-Galactic centre LOS. However, a comparison of the rotation and velocity dispersion curves at different heights from the plane, used by Zoccali et al. (2014) to obtain the rotation maps, are very similar to those of the bulge of NGC 4710. These 1D rotation profiles are also in good agreement with the simulation of a galaxy hosting a pure BP bulge. The differences identified between the inner regions of the $\sigma$ maps of the Milky Way and NGC 4710 are not seen in the 1D curves. This suggests that the vertical elongation observed in the central region of the 
$\sigma$ map of the Milky Way bulge could be an artefact from the plane interpolation method to account for the $\sigma$ peak observed at $b=-2$. Instead, the $\sigma$ peak in the Milky Way bulge would be limited to Galactic latitudes $|b|<2(\sim 0.28 \mathrm{kpc})$.

On the other hand, the vertical metallicity gradient of $0.35 \mathrm{dex} / \mathrm{kpc}$ measured in the BP bulge of NGC $4710 \mathrm{com}-$ pares well to the vertical metallicity gradient measured in the Milky Way bulge via photometric (Gonzalez et al. 2013) and spectroscopic (Zoccali et al. 2008; Rojas-Arriagada et al. 2014; Ness et al. 2013) surveys of $\sim 0.40 \mathrm{dex} / \mathrm{kpc}$.

In the Milky Way, there is significant evidence showing that the oldest bulge stars do not follow the bar and BP bulge spatial distribution. On the other hand, no spheroidal bulge component is found when performing an integrated light decomposition analysis of NGC 4710, thus this bulge is classified as a pure BP bulge. If present in NGC 4710, a spheroidal bulge component would have to be much less dominant than the BP to remain hidden from the light decomposition analysis. Despite this, our results show that the global chemodynamical properties of the bulge of NGC 4710, based on its integrated spectrum, are in excellent agreement with those of the resolved stellar populations of the Milky Way bulge, thus suggesting that these properties, i.e. the observed kinematic profiles and vertical metallicity gradient, are intrinsic to their BP bulge component. Certainly, the ongoing and planned surveys based on multi-object spectroscopic facilities with high multiplex capabilities (APOGEE, 4MOST, and MOONS) where a large number of metal-poor stars can be mapped, might hold the key to understand the nature of the oldest, most metal-poor stars of the Galactic bulge.

This study compares the global kinematics and morphological properties of the Milky Way bulge to an external galaxy. This work is demonstrative of the potential to understand our own galaxy in context via comparisons of its detailed stellar populations and kinematics from the rapidly growing large coverage spectroscopic and photometric datasets with those of other galaxies observed with IFU instruments, such as MUSE.

Acknowledgements. We are grateful for the useful comments received from the anonymous referee. We warmly thank the ESO Paranal Observatory staff for performing the observations for this programme. M.Z. and D.M. acknowledge funding from the BASAL CATA through grant PFB-06, and the Chilean Ministry of Economy through ICM grant to the Millennium Institute of Astrophysics. M.Z. acknowledges support by Proyecto Fondecyt Regular 1150345. Support for this project is provided by CONICYT's PCI programme through grant DPI20140066. D.M. acknowledges support by FONDECYT No. 1130196. V.P.D. is supported by STFC Consolidated grant \# ST/J001341/1. The simulation used in this study was run at the High Performance Computer Facility of the University of Central Lancashire.

\section{References}

Athanassoula, E. 2005, MNRAS, 358, 1477

Athanassoula, E. 2008, in Mapping the Galaxy and Nearby Galaxies, eds.

K. Wada, \& F. Combes (Springer), Astrophys. Space Sci. Proc., 47

Athanassoula, E., \& Misiriotis, A. 2002, MNRAS, 330, 35

Babusiaux, C., Gómez, A., Hill, V., et al. 2010, A\&A, 519, A77

Bacon, R., Accardo, M., Adjali, L., et al. 2010, in SPIE Conf. Ser., 7735, 773508

Bekki, K., \& Tsujimoto, T. 2011, MNRAS, 416, L60

Bensby, T., Yee, J. C., Feltzing, S., et al. 2013, A\&A, 549, A147

Brooks, A., \& Christensen, C. 2016, in Galactic Bulges, eds. E. Laurikainen, R. Peletier, \& D. Gadotti (Springer International Publishing), Astrophys. Space Sci. Lib., 418, 317
Bureau, M., Aronica, G., Athanassoula, E., et al. 2006, MNRAS, 370, 753

Buta, R. J., Sheth, K., Athanassoula, E., et al. 2015, ApJS, 217, 32

Cappellari, M., \& Copin, Y. 2003, MNRAS, 342, 345

Cappellari, M., \& Emsellem, E. 2004, PASP, 116, 138

Cappellari, M., McDermid, R. M., Alatalo, K., et al. 2013, MNRAS, 432, 1862

Cole, D. R., Debattista, V. P., Erwin, P., Earp, S. W. F., \& Roškar, R. 2014, MNRAS, 445, 3352

Combes, F., \& Sanders, R. H. 1981, A\&A, 96, 164

Combes, F., Debbasch, F., Friedli, D., \& Pfenniger, D. 1990, A\&A, 233, 82

Davis, T. A., Alatalo, K., Sarzi, M., et al. 2011, MNRAS, 417, 882

de Souza, R. E., Gadotti, D. A., \& dos Anjos, S. 2004, ApJS, 153, 411

Dékány, I., Minniti, D., Catelan, M., et al. 2013, ApJ, 776, L19

Dékány, I., Minniti, D., Majaess, D., et al. 2015, ApJ, 812, L29

Gadotti, D. A. 2008, MNRAS, 384, 420

Gadotti, D. A. 2012, Astron. Astrophys. Trans., 27, 221

Gadotti, D. A., Seidel, M. K., Sánchez-Blázquez, P., et al. 2015, A\&A, 584, A90

Gonzalez, O., \& Gadotti, D. 2016, in Galactic Bulges, eds. E. Laurikainen,

R. Peletier, \& D. Gadotti (Springer International Publishing), Astrophys. Space Sci. Lib., 418, 199

Gonzalez, O. A., Rejkuba, M., Zoccali, M., et al. 2012, A\&A, 543, A13

Gonzalez, O. A., Rejkuba, M., Zoccali, M., et al. 2013, A\&A, 552, A110

Hill, V., Lecureur, A., Gómez, A., et al. 2011, A\&A, 534, A80

Howard, C. D., Rich, R. M., Clarkson, W., et al. 2009, ApJ, 702, L153

Iannuzzi, F., \& Athanassoula, E. 2015, MNRAS, 450, 2514

Johnson, C. I., Rich, R. M., Kobayashi, C., et al. 2013, ApJ, 765, 157

Kormendy, J. 2013, Secular Evolution in Disk Galaxies, eds. J. Falcón-Barroso, \& J. H. Knapen, 1

Krajnović, D., Cappellari, M., de Zeeuw, P. T., \& Copin, Y. 2006, MNRAS, 366, 787

Krajnović, D., Karick, A. M., Davies, R. L., et al. 2013, MNRAS, 433, 2812

Laurikainen, E., \& Salo, H. 2016, in Galactic Bulges, eds. E. Laurikainen, R. Peletier, \& D. Gadotti (Springer International Publishing), Astrophys. Space Sci. Lib., 418, 77

Laurikainen, E., Salo, H., \& Buta, R. 2005, MNRAS, 362, 1319

Laurikainen, E., Salo, H., Buta, R., Knapen, J. H., \& Comerón, S. 2010, MNRAS, 405, 1089

Lütticke, R., Dettmar, R.-J., \& Pohlen, M. 2000, A\&AS, 145, 405

Martinez-Valpuesta, I., \& Gerhard, O. 2013, ApJ, 766, L3

McWilliam, A., \& Zoccali, M. 2010, ApJ, 724, 1491

McWilliam, A., Fulbright, J., \& Rich, R. M. 2010, in IAU Symp. 265, eds. K. Cunha, M. Spite, \& B. Barbuy, 279

Méndez-Abreu, J., Debattista, V. P., Corsini, E. M., \& Aguerri, J. A. L. 2014, A\&A, 572, A25

Minniti, D., Lucas, P. W., Emerson, J. P., et al. 2010, New Astron., 15, 433

Nataf, D. M., Udalski, A., Gould, A., Fouqué, P., \& Stanek, K. Z. 2010, ApJ, 721, L28

Ness, M., Freeman, K., Athanassoula, E., et al. 2013, MNRAS, 430, 836

Ness, M., Debattista, V. P., Bensby, T., et al. 2014, ApJ, 787, L19

Pietrukowicz, P., Kozłowski, S., Skowron, J., et al. 2015, ApJ, 811, 113

Rojas-Arriagada, A., Recio-Blanco, A., Hill, V., et al. 2014, A\&A, 569, A103

Saha, K., \& Gerhard, O. 2013, MNRAS, 430, 2039

Saito, R. K., Zoccali, M., McWilliam, A., et al. 2011, AJ, 142, 76

Scott, N., Cappellari, M., Davies, R. L., et al. 2013, MNRAS, 432, 1894

Shen, J., Rich, R. M., Kormendy, J., et al. 2010, ApJ, 720, L72

Sheth, K., Melbourne, J., Elmegreen, D. M., et al. 2012, ApJ, 758, 136

Simmons, B. D., Melvin, T., Lintott, C., et al. 2014, MNRAS, 445, 3466

Stanek, K. Z., Mateo, M., Udalski, A., et al. 1994, ApJ, 429, L73

Valenti, E., Zoccali, M., Renzini, A., et al. 2013, A\&A, 559, A98

Valenti, E., Zoccali, M., Gonzalez, O. A., et al. 2016, A\&A, 587, L6

Vásquez, S., Zoccali, M., Hill, V., et al. 2013, A\&A, 555, A91

Vazdekis, A., Sánchez-Blázquez, P., Falcón-Barroso, J., et al. 2010, MNRAS, 404, 1639

Wegg, C., \& Gerhard, O. 2013, MNRAS, 435, 1874

Wegg, C., Gerhard, O., \& Portail, M. 2015, MNRAS, 450, 4050

Williams, M. J., Zamojski, M. A., Bureau, M., et al. 2011, MNRAS, 414, 2163

Young, L. M., Bureau, M., Davis, T. A., et al. 2011, MNRAS, 414, 940

Zoccali, M. 2010, in IAU Symp. 265, eds. K. Cunha, M. Spite, \& B. Barbuy, 271

Zoccali, M., Renzini, A., Ortolani, S., et al. 2003, A\&A, 399, 931

Zoccali, M., Hill, V., Lecureur, A., et al. 2008, A\&A, 486, 177

Zoccali, M., Gonzalez, O. A., Vasquez, S., et al. 2014, A\&A, 562, A66 